\section{AUTHOR INDEX \\ VOLUME 47, 2005}

Anderson A. Comparative Radiocarbon Dating of Lignite, Pottery, and Charcoal Samples from Babeldaob Island, Republic of Palau, 1; See also Higham T, 367 Andrič M. See Goslar T, 115

Angulo RJ. Reservoir Effect of the Southern and Southeastern Brazilian Coast, 67

Ascough PL. Influence of Mollusk Species on Marine $\Delta R$ Determinations, 433

Barbetti M. See Jull AJT, vii(2)

Battipaglia G. See Marzaioli F, 257

Blau S. AMS Radiocarbon Dates of Kurgans Located on the Ust'-Yurt Plateau, Uzbekistan, 235

Boaretto E. Dating the Iron Age I/II Transition in Israel: First Intercomparison Results, 39; See also Yizhaq M, 193

Braje TJ. Reassessing Human Settlement on the South Coast of San Miguel Island, California: The Use of ${ }^{14}$ C Dating as a Reconnaissance Tool, 11

Bronk Ramsey C. See Higham T, 367

Bruun S. Estimating Turnover of Soil Organic Carbon Fractions Based on Radiocarbon Measurements, 99

Bryant CL. See Ertunç T, 453

Calcagnile L. See Quarta G, 27; See also Quarta G, 207

Chappell J. See Anderson A, 1

Charman DJ. Chronologies for Recent Peat Deposits Using Wiggle-Matched Radiocarbon Ages: Problems with Old Carbon Contamination, 135

Clark G. See Anderson A, 1

Cohen I. See Yizhaq M, 193

Cole JE. See Guilderson TP, 57

Cook GT. See Ascough PL, 433

Cotrufo MF. See Marzaioli F, 257

Currie M. See Ertunç T, 453

Czernik J. See Goslar T, 115

Davis LG. See Hall R, 383

D'Elia M. See Quarta G, 27; See also Quarta G, 207

de Jong AFM. See Rutgers LV, 395

de Souza MC. See Angulo RJ, 67

Domínguez Rubio I. See McClung de Tapia E, 159

D’Onofrio A. See Marzaioli F, 257

Druffel ERM. See Hwang J, 75

Dugmore AJ. See Ascough PL, 433

Eglinton TI. See Ohkouchi N, 401; See also Mollenhauer G, 413; See also Ohkouchi N, 425

Erlandson JM. See Braje TJ, 11

Ertunç T. Progress in AMS Target Production of Submilligram Samples at the NERC Radiocarbon Laboratory, 453

Fairbairn A. Re-dating Mid-Holocene Betelnut (Areca
Catechu L.) and Other Plant Use at Dongan, Papua New Guinea, 377

Fallick AE. See Hardie SML, 441

Fillmore M. See Hall R, 383

Freeman SPHT. See Ascough PL, 433; See also Ertunç T, 453

Gama Castro J. See McClung de Tapia E, 159

Gao S. See Guo Z, 221

Garnett MH. See Charman DJ, 135; See also Hardie SML, 441

Gerlach SE. See Reuther JD, 359

Gilboa A. See Boaretto E, 39

Goslar E. See Goslar T, 115

Goslar T. Radiocarbon Dating of Modern Peat Profiles: Pre-and Post-Bomb ${ }^{14} \mathrm{C}$ Variations in the Construction of Age-Depth Models, 115

Green R. See Petchey F, 181

Guilderson TP. Pre-Bomb $\Delta^{14} C$ Variability and the Suess Effect in Cariaco Basin Surface Waters as Recorded in Hermatypic Corals, 57; See also Kennedy MA, 265

Guo Z. AMS Radiocarbon Dating of the Fengxi Site in Shaanxi, China, 221; See also Liu K, 21

Hall R. Radiocarbon, Soil, and Artifact Chronologies for an Early Southern Oregon Coastal Site, 383

Han B. See Liu K, 21

Hardie SML. Carbon Dioxide Capture Using a Zeolite Molecular Sieve Sampling System for Isotopic Studies $\left({ }^{13} \mathrm{C}\right.$ and $\left.{ }^{14} \mathrm{C}\right)$ of Respiration, 441

Haynes V. See Jull AJT, vii(2)

Hicks S. See Goslar T, 115

Higham T. Diet-derived Variations in Radiocarbon and Stable Isotopes: A Case Study from Shag River Mouth, New Zealand, 367

Hughen KA. See Ohkouchi N, 425

Hwang J. Blank Correction for $\Delta^{14} C$ Measurements in Organic Compound Classes of Oceanic Particulate Matter, 75

Hyötylä H. See Goslar T, 115

Imamura M. See Yoshikawa H, 211

Ingravallo E. See Quarta G, 207

Jensen LS. See Bruun S, 99

Jull AJT. From the Editor, v(1); From the Editor, v(2); Paul Edward Damon Obituary, vii(2); From the Editor, v(3); See also Boaretto E, 39

Keigwin LD. See Ohkouchi N, 425

Kennedy MA. A Radiocarbon Chronology of HunterGatherer Occupation from Bodega Bay, California, USA, 265

Khalaily H. See Yizhaq M, 193 
Kim J-C. See Vasil'chuk A, 243

Kobayashi K. See Yoshikawa K, 211

Kromer B. See Usoskin IG, 31

Kutschera W. See Liu K, 21

Li K. See Guo Z, 221

Liston J. An Assessment of Radiocarbon Dates from Palau, Western Micronesia, 295

Liu K. AMS Radiocarbon Dating of Bone Samples from the Xinzhai Site in China, 21; See also Guo Z, 221

Lu X. See Guo Z, 221

Lubritto C. See Marzaioli F, 257

Ma H. See Liu K, 21; See also Guo Z, 221

Maden C. See Ertunç T, 453

Manzo A. The Stratigraphic Sequence at Yalâ (Yemen): A Statistical Evaluation, 147

Martin P. Book Review, 355

Marzaioli F. Reconstruction of Past $\mathrm{CO}_{2}$ Concentration at a Natural $\mathrm{CO}_{2}$ Vent Site Using Radiocarbon Dating of Tree Rings, 257

McClung de Tapia E. Radiocarbon Dates from Soil Profiles in the Teotihuacán Valley, Mexico: Indicators of Geomorphological Processes, 159

Miglietta F. See Marzaioli F, 257

Mintz G. See Yizhaq M, 193

Mollenhauer G. Radiocarbon Dating of Alkenones from Marine Sediments: II. Assessment of Carbon Process Blanks, 413

Montluçon D. See Ohkouchi N, 401; See also Mollenhauer G, 413

Murray C. See Ertunç T, 453

Nakahara H. See Yoshikawa H, 211

Nakanishi T. See Yoshikawa H, 211

Norton GA. Improved Tube Cracker for Opening Vacuum-Sealed Glass Tubes, 177

Ohkouchi N. Radiocarbon Dating of Alkenones from Marine Sediments: I. Isolation Protocol, 401; Radiocarbon Dating of Alkenones from Marine Sediments: III. Influence of Solvent Extraction Procedures on ${ }^{14} \mathrm{C}$ Measurements of Foraminifera, 425

Ostle NJ. See Hardie SML, 441

Passariello I. See Marzaioli F, 257

Paustian K. See Bruun S, 99

Petchey F. Use of Three Isotopes to Calibrate Human Bone Radiocarbon Determinations from Kainapirina (SAC), Watom Island, Papua New Guinea, 181

Phear S. See Anderson A, 1

Priller A. See Liu K, 21

Quarta G. New Bomb Pulse Radiocarbon Records from Annual Tree Rings in the Northern Hemisphere Temperate Region, 27; The Neolithic Site of Serra Cicora: Results of the AMS Radiocarbon Dating, 207
Räsänen S. See Goslar T, 115

Reddy CM. See Ohkouchi N, 401

Reimer PJ. See Angulo RJ, 67

Reuther JD. Testing the "Dicarb Problem": A Case Study from North Alaska, 359

Rick TC. See Braje TJ, 11

Rogalla D. See Marzaioli F, 257

Roosen E. See Ohkouchi N, 425

Rowland AP. See Hardie SML, 441

Rubino M. See Marzaioli F, 257

Russell AD. See Kennedy MA, 265

Rutgers LV. Radiocarbon Dates from the Catacombs of St. Callixtus in Rome, 395

Sasaoka SK. See Angulo RJ, 67

Scott EM. See Ascough PL, 433

Sedov S. See McClung de Tapia E, 159

Sharon I. See Boaretto E, 39

Six J. See Bruun S, 99

Solleiro E. See McClung de Tapia E, 159

Southon JR. See Guilderson TP, 57

Steier P. See Liu K, 21

Strumia S. See Marzaioli F, 257

Swadling P. See Fairbairn A, 377

Terrasi F. See Marzaioli F, 257

Theodórsson P. A Simple, Extremely Stable Single-Tube LS System for ${ }^{14} C$ Dating, 89; Simultaneously Measuring ${ }^{14} \mathrm{C}$ and Radon in Benzene Dating Samples, 231

Tiberi I. See Quarta G, 207

Tompkins C. See Higham T, 367

Usoskin IG. Reconstruction of the ${ }^{14} \mathrm{C}$ Production Rate from Measured Relative Abundance, 31

Valzano D. See Quarta G, 27

van der Borg K. See Rutgers LV, 395

van der Knaap WO. See Goslar T, 115

Vasil'chuk A. AMS ${ }^{14} \mathrm{C}$ Dating of Pollen Concentrate from Late Pleistocence Ice Wedges from the Bison and Seyaha Sites in Siberia, 243

Vasil'chuk Y. See Vasil'chuk A, 243

Wang J. See Guo Z, 221

Weiner S. See Yizhaq M, 193

Wild EM. See Liu K, 21

Willis S. See Hall R, 383

Wu X. See Liu K, 21; See also Guo Z, 221

Xu Li. See also Ohkouchi N, 401

Xu Lianggao. See Guo Z, 221

Xu S. See Ertunç T, 453

Yagodin V. See Blau S, 235

Yizhaq M. Quality Controlled Radiocarbon Dating of Bones and Charcoal from the Early Pre-Pottery 
Neolithic B (PPNB) of Motza (Israel), 193

Yoshikawa H. Determination of ${ }^{14} \mathrm{C}$ in Volcanic Gas by

Accelerator Mass Spectrometry, 211
Yuan S. See Liu K, 21; See also Guo Z, 221

Zhao C. See Liu K, 21 